\title{
ARTICLE OPEN \\ Spin splitting and electric field modulated electron-hole pockets in antimonene nanoribbons
}

\author{
Yan Song ${ }^{1}$, Xiaocha Wang ${ }^{2}$ and Wenbo $\mathrm{Mi}^{1}$
}

The one-dimensional nanoribbons have great potential applications in novel multifunctional electronic devices. Herewith, we investigate the electronic structure of antimonene nanoribbons using the first-principles calculations. Four ribbon models are considered. All of them are semiconductors with appropriate band gaps. Because of the loss of inversion symmetry, spin-orbit splitting appears in the asymmetric washboard nanoribbons. Significantly, spin splittings of 62 and 44 meV are found at the valence band maximum and conduction band minimum for zigzag asymmetric washboard nanoribbon (aW-zSbNR). Further applying an inplane electric field introduces a potential difference between the two zigzag edges. The energies for the localized edge states are modified. Eventually spin-orbit-coupling induced band inversion and electron-hole pockets with appropriately the same size could be triggered in a W-zSbNR, which is interesting for exploiting the magnetoresistance effect.

npj Quantum Materials (2017)2:15; doi:10.1038/s41535-017-0022-y

\section{INTRODUCTION}

Group 15 monolayers become another kind of attractive twodimensional (2D) materials after graphene ${ }^{1,2}$ hexagonal $\mathrm{BN}_{1}^{3-6}$ and transition metal dichalcogenides, ${ }^{7-11}$ due to their appropriate band gap and high carrier mobility. ${ }^{12}$ Zhang et al. ${ }^{12}$ predicted that the group 15 monolayers, namely phosphorene, arsenene, antimonene, and bismuthene, exhibit high thermodynamic stability and show a broad range of band gap from 0.36 to $2.62 \mathrm{eV}$. Significantly, phosphorene, arsenene, and bismuthene possess high carrier mobility of several thousand $\mathrm{cm}^{2} / \mathrm{V} / \mathrm{s}$, which is highly favored for nanoelectronics and optoelectronics.

The intriguing behavior of 2D monolayers is that they can be etched or carved into one-dimensional (1D) nanoribbons along a specific direction. With quantum confinement effect, nanoribbons exhibit many extraordinary electronic, ${ }^{13-15}$ optical $^{16}$ and magnetic properties. ${ }^{17,18}$ Half-metallicity was predicted in zigzag graphene nanoribbons by applying an in-plane electric field across the zigzag-shaped edges. ${ }^{19}$ Dimensional reduction facilitates the manipulation of electronic and magnetic properties by modifying the edge states. Decorating the edges with different chemical groups can make half-metallicity appear in zigzag graphene, ${ }^{20}$ boron nitride, ${ }^{21}$ and $\mathrm{MoS}_{2}$ nanoribbons. ${ }^{22}$

Very recently, multilayer arsenene and antimonene nanoribbons are successfully synthesized using plasma-assisted process. Controllable thickness and room-temperature orange light emission are realized, respectively. ${ }^{23,24}$ Experimental breakthroughs in fabricating nanoribbons convince the bright future of 1D structure in nanoelectronic applications. In this work, we simulate the electronic properties of antimonene nanoribbons with two stable structures predicted in previous studies. ${ }^{25}$ Considering that the spin-orbit coupling (SOC) in heavy element Sb is significant, we include the SOC explicitly in our calculations. It is found that the intrinsic SOC in asymmetric washboard nanoribbons removes the spin degeneracy. Applying an in-plane electric field in a specific model can realize the electron-hole-pocket structure, which is attractive for studying the magnetoresistance (MR) effect in these nanoribbons. ${ }^{26}$

\section{RESULTS}

We construct antimonene nanoribbons within two stable structures: buckled honeycomb and asymmetric washboard structures, using the names adopted in ref. 25. The armchair and zigzag edge geometries are considered. The structures of four nanoribbon models are shown in Fig. 1. Comparing the balance of edge coupling and consumption of computations, we choose nine rows of atoms for asymmetric washboard armchair antimonene nanoribbon (aW-aSbNR), asymmetric washboard zigzag antimonene nanoribbon (aW-zSbNR), buckled honeycomb armchair antimonene nanoribbon (B-aSbNR), and six rows of atoms for buckled honeycomb zigzag antimonene nanoribbon (B-zSbNR) (Fig. 1) as the ribbon width. The asymmetric washboard antimonene (Fig. 1a, b) contains two parallel atomic planes and presents a rectangular lattice similar to phosphorene. The primitive unit cell contains four $\mathrm{Sb}$ atoms. Each $\mathrm{Sb}$ is threefold coordinated with in-plane and inter-planar $\mathrm{Sb}$ atoms. Different from phosphorene, one of the two $\mathrm{Sb}$ atoms located in either plane in the primitive cell slightly displaces outwards to prevent the structure from long-wave instability, ${ }^{25}$ as seen in the side view of Fig. $1 \mathrm{~b}$.

Another stable structure is the buckled honeycomb structure (Fig. 1c, d) related to the bulk layered $\mathrm{Sb}$ with a space group of $R 3-$ $m h$. In hexagonal lattice, the $s p^{2}$ bonds of the unstable planar honeycomb structure rehybridize to maintain the stability through buckling, ${ }^{25}$ as seen in the side view of Fig.1d. Zhang et al. ${ }^{12}$ demonstrated that antimonene with these two structures exhibit thermodynamic stability. The energy of the buckled honeycomb structure is $14 \mathrm{meV} / \mathrm{atom}$ lower than that of asymmetric washboard one. ${ }^{12}$

\footnotetext{
${ }^{1}$ Tianjin Key Laboratory of Low Dimensional Materials Physics and Preparation Technology, School of Science, Tianjin University, Tianjin 300354, China and ${ }^{2}$ School of Electronics Information Engineering, Tianjin University of Technology, Tianjin 300384, China

Correspondence: Wenbo Mi (miwenbo@tju.edu.cn)
}

Received: 11 November 2016 Revised: 2 February 2017 Accepted: 1 March 2017

Published online: 17 March 2017 
(a) aW-aSbNR

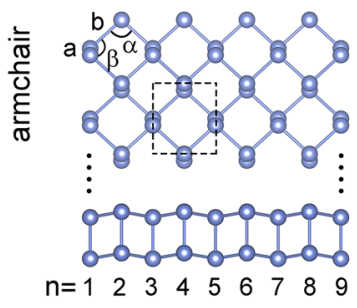

(c) B-aSbNR

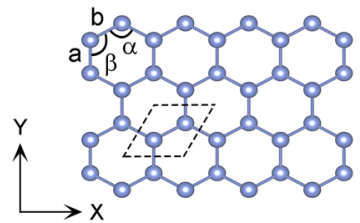

z 8888888888 $\mathrm{n}=1 \quad 2 \quad 3 \quad 4 \quad 5 \quad 6 \quad 7 \quad 8 \quad 9$ $\rightarrow \mathrm{x}$ (b) aW-zSbNR
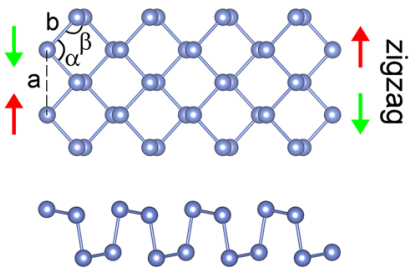

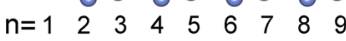

(d) B-zSbNR
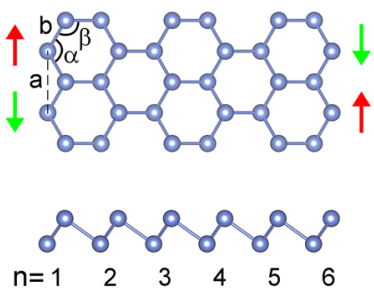

Fig. 1 Top and side views of a aW-aSbNR, $\mathbf{b}$ aW-zSbNR, c B-aSbNR, and $\mathbf{d}$ B-zSbNR. Initialization of edge magnetism is depicted by green (spin down) and red (spin up) arrows

We construct nanoribbons along two typical directions: armchair and zigzag directions. The width of each model is depicted in Fig. 1. In ref. 27, the spin density wave state was predicted to be the ground state in zigzag phosphorene, arsenene, and antimonene nanoribbons over the charge density wave state. Hence, we adopt the intra- and inter-edge antiferromagnetic (AFM) orders for the zigzag nanoribbons. ${ }^{27}$ The initialization of the edge magnetism is depicted in Fig. 1b, d. The structure optimizations are performed using the conjugate gradient algorithm. We characterize the optimized structures by the relevant structural parameters including the bond angels $(\alpha, \beta)$ and bond lengths $(a, b)$ (Fig. 1), as listed in Table 1. For zigzag nanoribbons, spin density wave is indeed observed. The spin density differences for aW-zSbNR and B-zSbNR are plotted in Fig. 2a, b, with red (green) labels for the spin up (spin down) density with the isosurface value of $0.001 \mathrm{e} / \AA$. The edge atoms are coupled antiferromagnetically. For aW-zSbNR, the spin distribution of the edge atoms shows the $p_{x}$ and $p_{z}$ characteristic. In B-zSbNR, the spin distribution shows the $p_{x}$ characteristic. The magnetic moments of the atoms marked in Fig. 2a, b are given in Table 1. The magnetic moments of edge atoms in a W-zSbNR are -0.10 and $0.08 \mu_{\mathrm{B}}$. In ref. 27 , the edge magnetic moment of zigzag arsenene nanoribbon calculated using Perdew-Burke-Ernzerhof (PBE) method is $0.07 \mu_{\mathrm{B}}$. In B-ZSbNR, it is $0.11 \mu_{\mathrm{B}}$. For the two armchair nanoribbons, they are both indirect-band-gap

Table 1. Values of relevant structural parameters $a, b, a$, and $\beta$ in Fig. 1 and magnetic moments of Sb1 Sb4 atoms in Fig. $2 a$, b

\begin{tabular}{|c|c|c|c|c|c|c|c|c|}
\hline & \multicolumn{4}{|c|}{ Lattice parameters ( $\AA /$ deg) } & \multicolumn{4}{|c|}{ Magnetic moments $\left(\mu_{\mathrm{B}}\right)$} \\
\hline aW-aSbNR & 2.79 & 2.91 & 86.91 & 86.12 & - & - & - & - \\
\hline aW-zSbNR & 4.27 & 2.89 & 95.20 & 74.02 & -0.10 & 0.04 & -0.04 & 0.08 \\
\hline B-aSbNR & 2.85 & 2.86 & 85.43 & 62.79 & - & - & - & - \\
\hline B-zSbNR & 4.05 & 2.84 & 90.97 & 77.09 & 0.11 & -0.04 & 0.04 & -0.11 \\
\hline
\end{tabular}

(a) aW-zSbNR
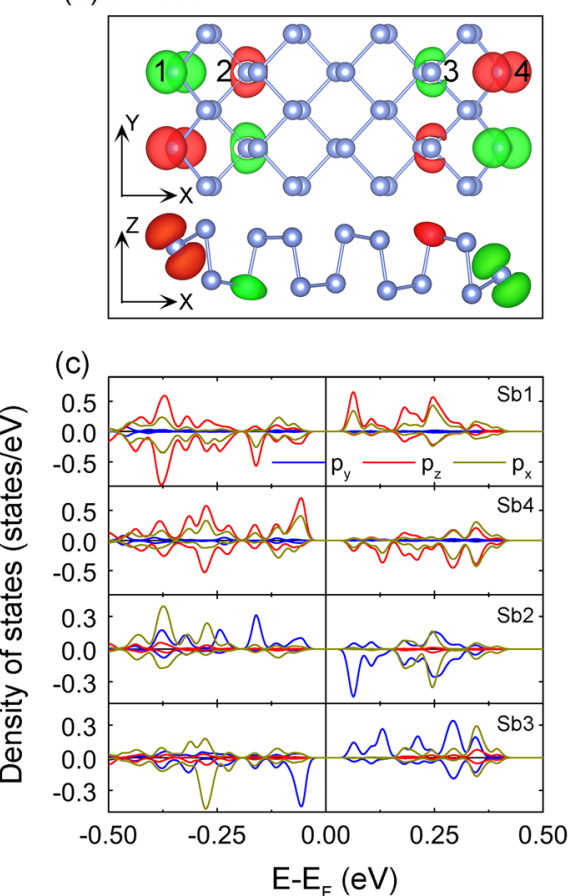

(b) B-zSbNR

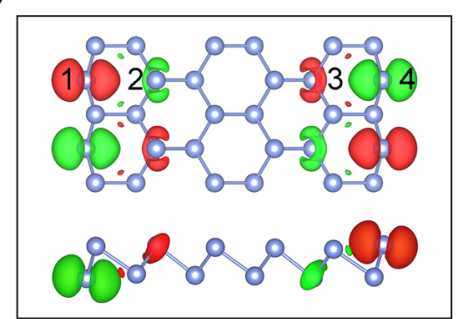

(d)

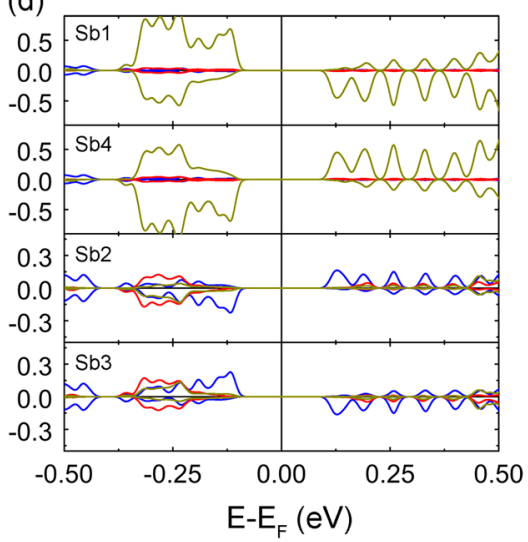

Fig. 2 Spatial spin density distribution $\left(\rho_{\uparrow}-\rho_{\downarrow}\right)$ for $\mathbf{a}$ aW-zSbNR and $\mathbf{b}$ B-zSbNR: spin up, red; spin down, green. The value of isosurface is 0.001 e/ $\AA^{3}$. The orbital-resolved DOS for Sb1 $\sim \mathrm{Sb} 4$ atoms in $\mathbf{a}$ and $\mathbf{b}$ are plotted in $\mathbf{c}$ and $\mathbf{d}$, respectively 


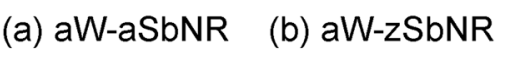
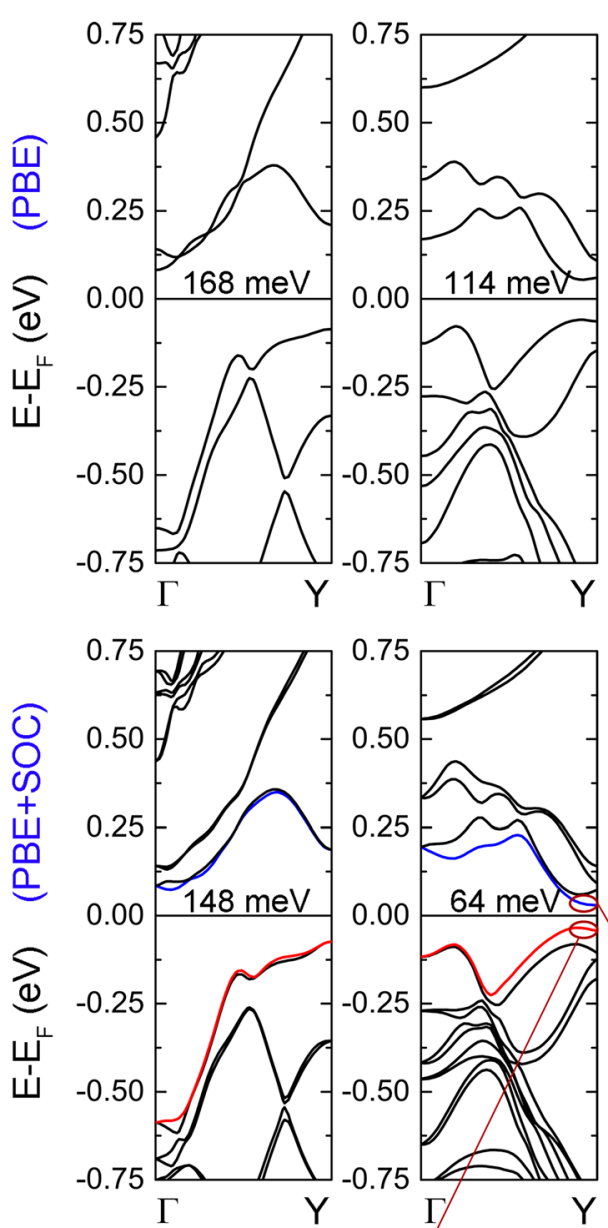

(e) VBM (c) B-aSbNR
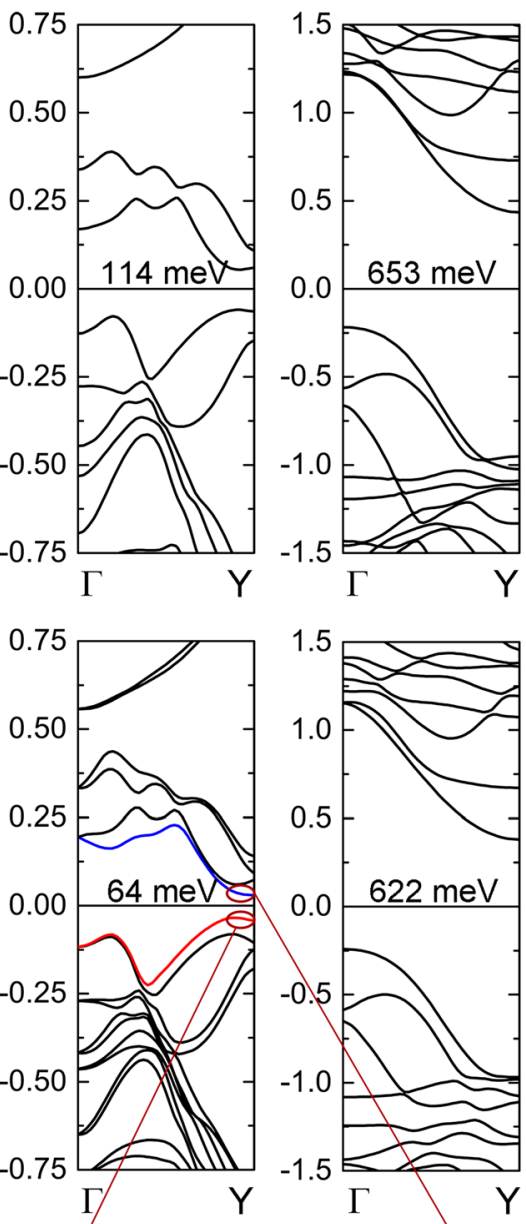

$-0.75 \frac{1}{1}$
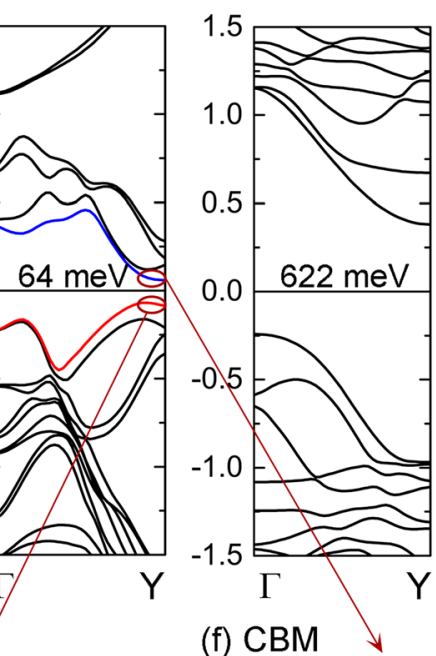

(d) B-zSbNR
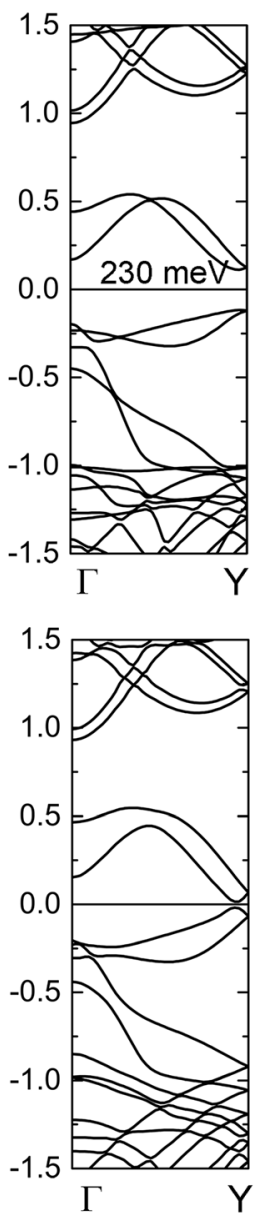
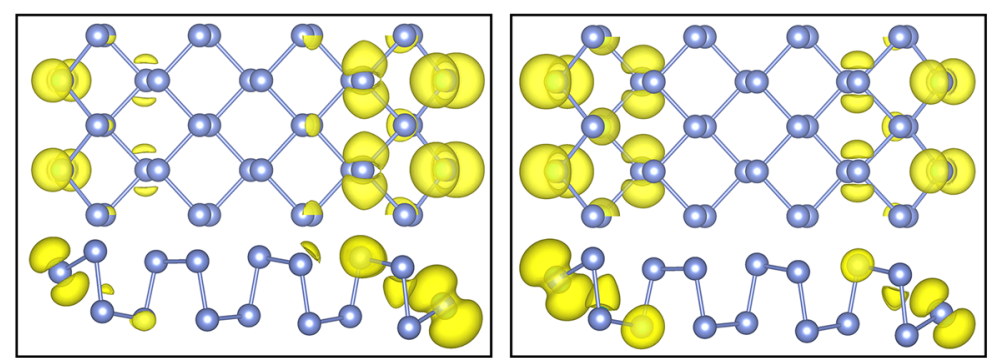

Fig. 3 Band structure of a aW-aSbNR, $\mathbf{b}$ aW-zSbNR, $\mathbf{c}$ B-aSbNR, and $\mathbf{d}$ B-zSbNR with and without SOC. The UVB and LCB of aW-aSbNR and aWzSbNR are highlighted by red and blue lines. Partial charge densities of e VBM and $\mathbf{f}$ CBM of aW-zSbNR. The isosurface value is 0.25 e/nm ${ }^{3}$

semiconductors as illustrated later. Hence there is no Peierls instability. Accordingly, no edge magnetism is observed in the armchair nanoribbons.

Figure 2c, d gives the orbital-resolved density of states (DOS) for Sb1-Sb4 of aW-zSbNR and B-zSbNR, respectively. As shown in Fig. 2c, the spin polarization of Sb1 and Sb4 comes from the $p_{z}$ and $p_{x}$ orbitals. For $\mathrm{Sb} 2$ and $\mathrm{Sb} 3$, the spin polarization is attributed to the $p_{x}$ and $p_{y}$ orbitals. In Fig. $2 d$, it is seen that the spin polarization of Sb1 and Sb4 mainly comes from $p_{x}$ orbitals and that of $\mathrm{Sb} 2$ and $\mathrm{Sb} 3$ mainly comes from $p_{y}$ orbitals. The spin polarization of the $p$ states in the valence band is consistent with the magnetic configurations of spin density difference shown in Fig. 2a, b.

The band structures of the four nanoribbons with and without SOC, respectively are shown in Fig. 3a-d. A comparison reveals several similarities: (1) No matter whether SOC is included or not, the zigzag nanoribbons are direct-band-gap semiconductors, while the armchair nanoribbons only have indirect band gaps; (2) SOC reduces the band gap in all the configurations, and the reductions of 20,50,31, and $190 \mathrm{meV}$ are induced in aW-aSbNR, aW-zSbNR, B-aSbNR, and B-zSbNR, respectively; (3) for the two asymmetric washboard nanoribbons, SOC lifts the spin degeneracy of the bands. More noticeable in a W-zSbNR is that the spin splittings at the valence band maximum (VBM) and conduction band minimum (CBM) are 62 and $44 \mathrm{meV}$, respectively. Hence, SOC cannot be neglected in simulating the electronic structure since it modifies the frontier states, especially in a W-zSbNR. It is worthy to be mentioned that the band structures of the four types of antimonene nanoribbons were investigated in ref. 25 . The ribbon width of aW-aSbNR, B-aSbNR, and B-zSbNR used to 
(a) aW-aSbNR

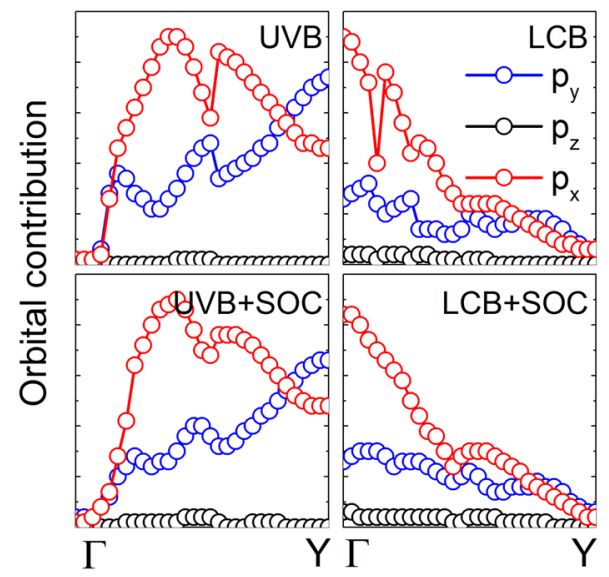

(c) B-aSbNR

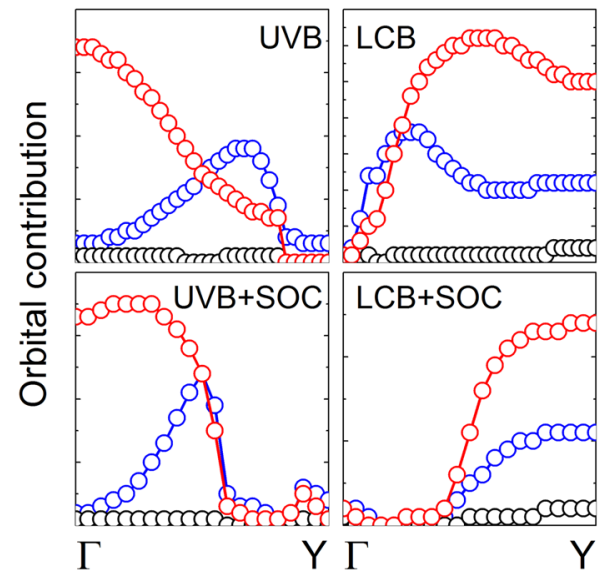

(b) aW-zSbNR

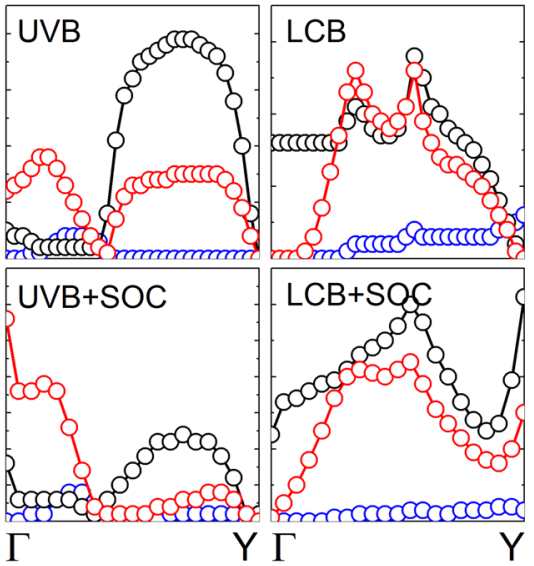

(d) B-zSbNR

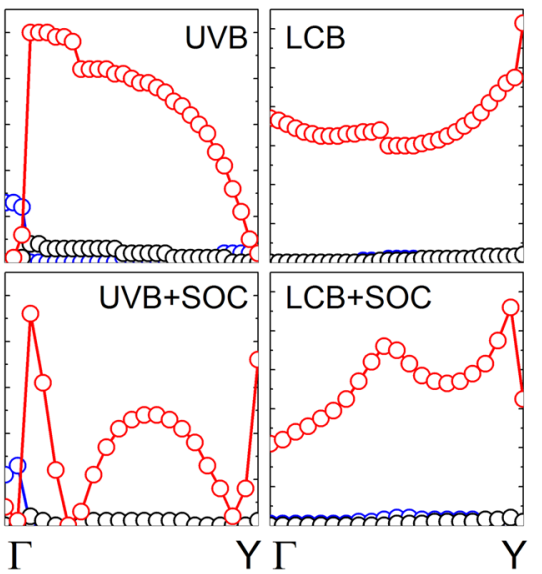

Fig. 4 Orbitally resolved contributions of the edge atoms to the UVB and LCB in arbitrary units for $\mathbf{a}$ aW-aSbNR, $\mathbf{b}$ aW-zSbNR, c B-aSbNR, and d B-zSbNR, respectively

calculate the band structure is different from our calculations. Especially, B-zSbNR was predicted to be a ferromagnetic (FM) semiconductor in ref. 25, while in our results the bands are spin-degenerate. The discrepancy is attributed to the different model adopted. And more, in our calculations, the initialization of the edge magnetism is chosen to be the AFM order. For comparison, the band structure for B-zSbNR with FM order is also calculated, and the structure is similar to that with the AFM order but the gap is $35 \mathrm{meV}$ smaller. More importantly, the total energy for the FM order is $8.8 \mathrm{meV}$ higher than that for the AFM order. It again demonstrates that the spin density wave is the ground state for the zigzag nanoribbons, consistent with earlier prediction in ref. 27.

An explanation of the SOC-induced band-gap reduction is provided by an investigation of the orbital contributions to the uppermost valance band (UVB) and lowermost conduction band (LCB) along the $\Gamma-Y$ line. For all the nanoribbons, the orbital contributions of all the atoms show no significant difference before and after an inclusion of SOC. The orbital contributions for the four nanoribbons with and without SO Care available in Fig. S1 in the Supplementary information. However, analysis of the partial charge densities at the edges of valance and conduction bands shows that the band edge states are localized at the edges of the nanoribbons. The partial charge densities for aW-zSbNR are shown in Fig. 3e, $f$ and the data for aW-aSbNR, B-aSbNR, and BzSbNR are given in Fig. S2 in the Supplementary information. The orbital contributions of the edge atoms are plotted in Fig. 4.
For aW-aSbNR, the UVB and LCB are contributed from the $p_{x}$ and $p_{y}$ states. SOC has little modification effect on these orbitals corresponding to the smallest band-gap reduction. SOC alters the contributions of the $p_{x}$ and $p_{z}$ states for aW-zSbNR and the $p_{x}$ and $p_{y}$ states for B-armchair. However, the general trends shown in these plots can still be recognized, in line with the moderate gap reductions. For $\mathrm{B}-\mathrm{zS}$ SbNR with the largest gap reduction, the UVB and LCB are contributed from the $p_{x}$ states. With turn-on of SOC, the contributions are changed significantly. Especially close to the $Y$ point at the UVB, the contribution is enhanced remarkably. Hence, it is suggested that the SOC-induced band-gap reduction is attributed to the modification on the orbital contributions of the localized edge states.

For the symmetry arguments, the absence of inversion symmetry in an asymmetric washboard structure is the reason that causes a spin-splitting of the energy bands. The symmetry of the asymmetric washboard antimonene is $P m n 2_{1}\left(C_{2 v}^{7}\right)$. A proper cutting of the nanoribbons aW-aSbNR and aW-zSbNR allows the symmetry reduction to $\mathrm{Pm}\left(C_{s}^{1}\right)$. The both cases are noncentrosymmetric. So SOC will break the spin-degeneracy along the $\Gamma-Y$ line (Fig. $3 a, b)$. On the contrary, the buckled antimonene and antimonene nanoribbons are centrosymmetric with the symmetry groups $P \overline{3} m 1\left(D_{3 d}^{3}\right)$ and $P 2 / m\left(C_{2 h}^{1}\right)$, respectively, which satisfy the inversion symmetry. Accordingly, the energy bands of B-aSbNR and B-zSbNR are spin-degenerate (Fig. 3c, d).

From Fig. $3 e, f$, it is seen that both the VBM and CBM are constructed from the edge atoms that present an AFM order 
(a)

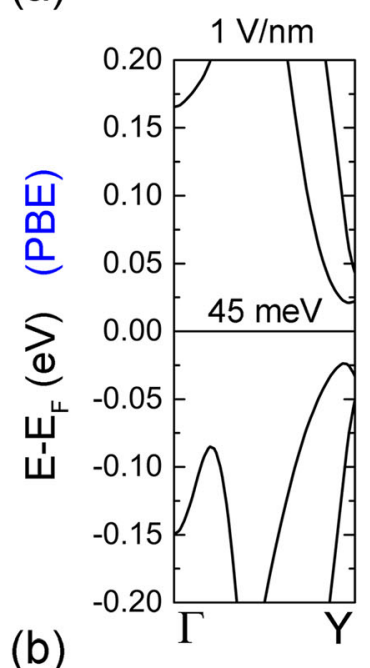

(b)

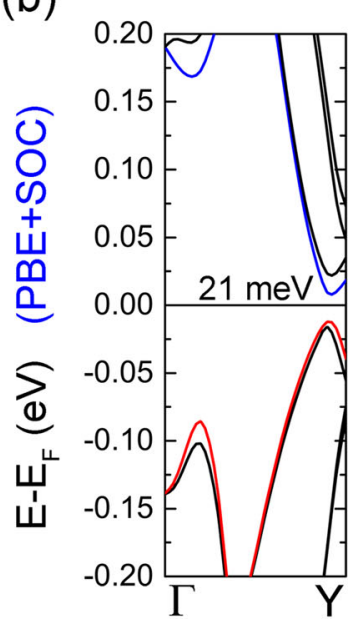

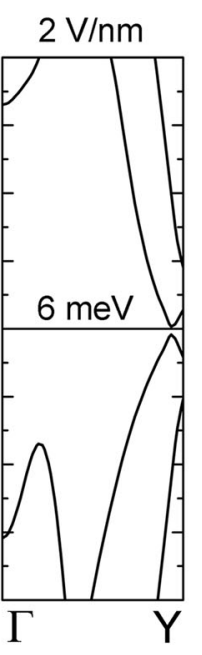

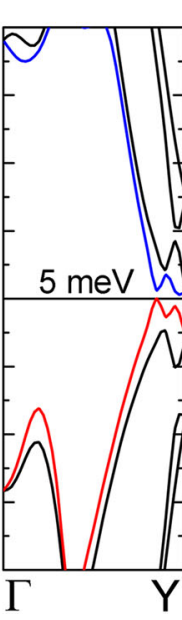

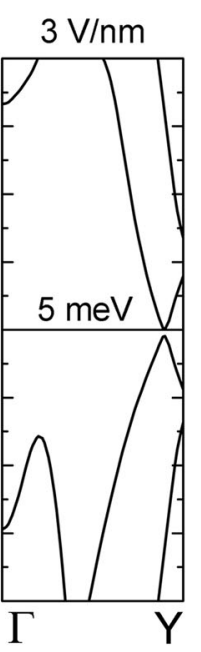
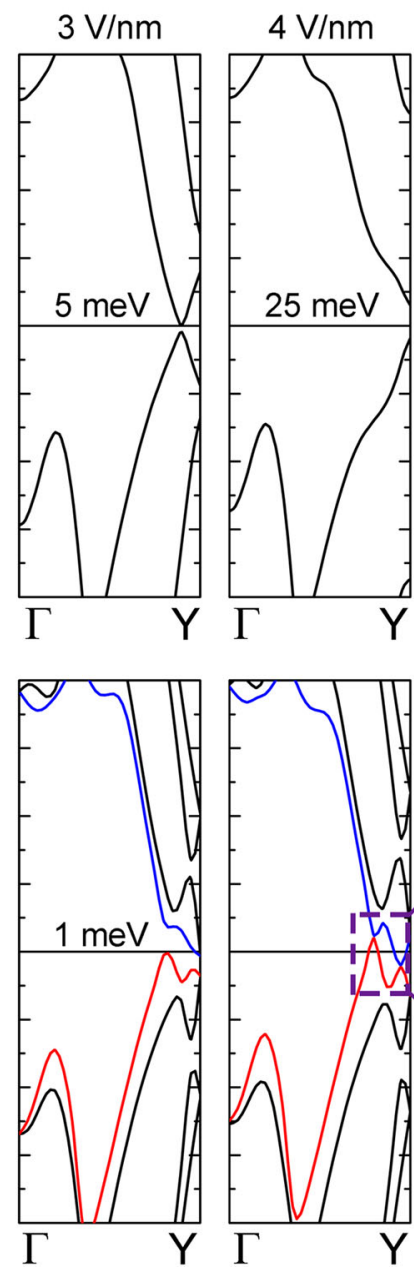

(c)

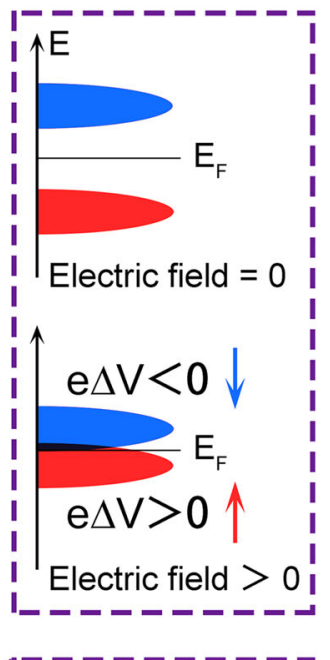

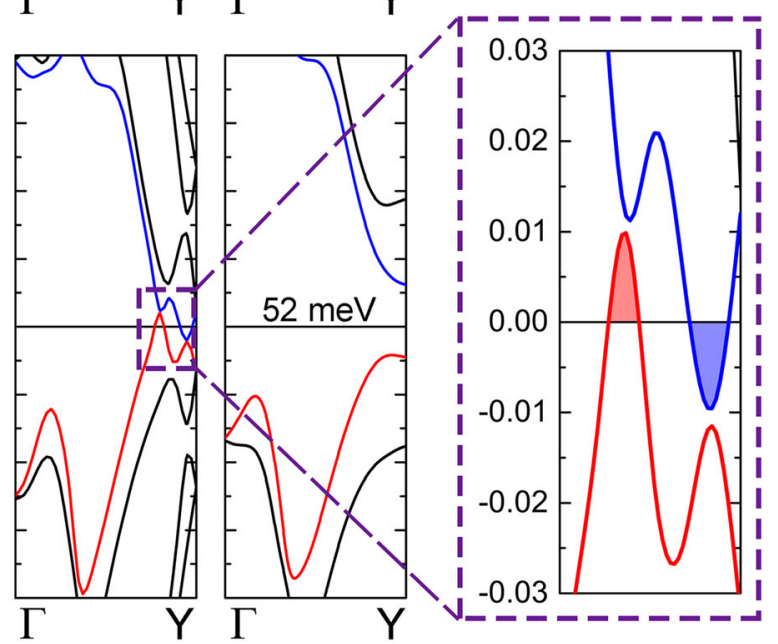

Fig. 5 Band structure of aW-zSbNR with respect to electric field without (a) and with (b) SOC. c Schematic DOS diagram of aW-zSbNR with and without an in-plane electric field

(Fig. 2a). It indicates that the splitting at the $Y$ point in a W-zSbNR is attributed to the AFM order that further reduces the symmetry at this point. Moreover, there exists an imbalance of charge distribution, whereas other nanoribbons do not have this property (Fig. S2). The VBM has more population at the right edge, while the CBM has more population at the left edge. The spatially separated edge states suggest that it is possible to modulate the energy bands around the Fermi level. ${ }^{19}$ Therefore, we apply an in-plane electric field across the edges of aW-zSbNR (along the $x$ direction in Fig. 1). The variations of band structure under electric field, without (a) and with (b) SOC are given in Fig. 5a, b, respectively. In case of no SOC, the band gap first decreases and then increases with the increasing field. When SOC is turned on, the twofold spin degeneracy is lifted. At a field of $2 \mathrm{~V} / \mathrm{nm}$, the bands around the VBM and CBM transform from the " $\wedge$ shape" to the "M shape". This variation is reminiscent of the band inversion, just like the case in 2D SbAs exposed to biaxial strain. ${ }^{28}$ Further increasing the electric field allows the appearance of electron and hole pockets with appropriately the same size at $4 \mathrm{~V} / \mathrm{nm}$. A clearer image can be seen in the zoom-in area. The semiconductor-semimetal transition originates from the fact that the electric field induces the energy-level shifts of opposite signs for the spatially separated band edge states. The electrostatic potential is raised on the right side and lowered on the left side as electric field increases. Because of the imbalanced distribution, the effects of electric field on them are opposite. Correspondingly, the energies for the localized edge states on the right side (mainly contributed to the VBM) are shifted upwards and those on the left side (mainly contributed to the CBM) downwards. The schematic DOS diagrams of aW-zSbNR with and without electric field are given in Fig. $5 c$. Further increasing the electric field can dismiss the electron-hole-pocket structure. It is well known that the PBE functional tends to underestimate the band gap, but the variation trend of bands that the VBM is shifted upwards while the CBM is shifted downwards by the electric field is valid. For example, in ref. 28, the topological transition in monolayer SbAs under applied strain is predicted using PBE method. Subsequently, the band structures at the hybrid functional level are given in the Supplementary Information. The results show that both the variation trend of bands and the topological properties are not influenced by the functional adopted. The gap closure occurs at a tensile strain of $12 \%$ at the PBE level $(14.8 \%$ at the Heyd-Scuseria-Ernzerhof (HSE) screened hybrid functional level). Given the mechanism proposed in Fig. $5 c$, it is also expected that the semiconductor-semimetal transition could be realized but merely under a strong electric field.

\section{DISCUSSION}

The electron-hole pockets are very attractive because of the extraordinary MR effect recently found in $\mathrm{WTe}_{2}$ (ref. 26). Extremely large-positive magnetoresistance (XMR) without any sign of saturation was reported at magnetic field up to $60 \mathrm{~T}$ (ref. 26). 
The MR is characterized by a near-quadratic field dependence and a field-induced up-turn in the resistivity, followed by a plateau at low temperature. ${ }^{29}$ Soon after, these characters were also observed in several semimetals. ${ }^{30-34}$ Different mechanisms have been proposed to explain the exotic quadratic XMR behaviors. ${ }^{26,30,33,35}$

Using high-resolution angle-resolved photoemission spectroscopy, Pletikosić et al. ${ }^{36}$ demonstrated that the tiny electron and hole pockets of equal size are the fundamental ingredients of physics for the unusual transport properties of $\mathrm{WTe}_{2}$. Cai et al. ${ }^{37}$ reported that the sizes of the electron-hole pockets show increased difference with pressure. The pressure dependent MR supports the perfect balance scenario. A recent study on the dopant effect in $\mathrm{WTe}_{2}$ also supported the electron-holecompensation theory. ${ }^{38}$ Nevertheless, a non-saturated XMR was found in compensated semimetal LaSb. ${ }^{29}$ The suppression of MR without observable change of the Fermi surfaces with increasing temperature was observed. ${ }^{29}$ This is contradictory to the above results. Moreover, recent studies showed deviations from the perfect electron-hole compensation in gated $\mathrm{WTe}_{2}$ thin flakes and $\mathrm{WTe}_{2}$ under magnetic field, whereas the XMR effects still exist. ${ }^{39,} 40$ Hence, so far the nature of the exotic XMR effect is still controversial.

Here, we report that the electric-field-modulated carrier compensation can be realized in a W-zSbNR. If the unsaturated $\mathrm{XMR}$ of $\mathrm{WTe}_{2}$ is attributed to this special resonance according to ref. 26 , the aW-zSbNR may serve as a platform for testing the cause of this extraordinary MR effect. In addition, aW-zSbNR has more similarities with the elemental materials graphite and bismuth, i.e., low carrier density, small effective mass, and an equal number of electrons and holes, which display XMR at low temperature. ${ }^{41}$ These features specific to semimetals give rise to the field-induced metal-insulator behavior. ${ }^{41}$ Thus, aW-zSbNR is attractive for exploring the positive MR effect. At high-magnetic field, the MR of graphite and bismuth saturates to a fieldindependent value. ${ }^{26}$ The unsaturated MR of $\mathrm{WTe}_{2}$ is attributed to the maintenance of the perfect charge compensation even at high field. ${ }^{26}$ The ratio between the $p$ - and $n$-type carriers becomes more and more severe with increasing magnetic field. A slight deviation will lead to the saturation of MR, which is the case of graphite and bismuth. ${ }^{26}$ Hence, it is suggested that the aW-zSbNR is particularly interested for studying the MR effect.

Besides, it is also demonstrated that the balance between the electron and hole states in $\mathrm{WTe}_{2}$ is established only beyond finite number of layers (three), showing that the behavior of $\mathrm{WTe}_{2}$ is not strictly two dimensional. ${ }^{42}$ Nevertheless, for nanoelectronics, it is desirable to obtain low-dimensional systems that have the performance as good as, or even better than, their bulk counterparts, favoring the potentials of antimonene nanoribbons.

A problem in the theory-oriented works ${ }^{19,43,44}$ on the electric field effects is that the involved electric field strength is relatively strong. It is still a great challenge to be realized in experimental research. Nevertheless, the electrically tunable property of antimonene nanoribbons is a good demonstration of the theoretical concept and does have implications for potential applications. This issue also stimulates further study of new principles or paradigms that are more feasible for achieving electric-field-tunable properties. Instead of applying strong external electric fields, using the built-in electric fields in materials or at the interfaces is another direction deserving further attention.

In summary, we have studied the electronic properties of antimonene nanoribbons by means of first-principles calculations. The spin density wave state is observed in the zigzag models. Because of the loss of inversion symmetry, SOC lifts the spin degeneracy for the asymmetric washboard nanoribbons. More remarkable in aW-zSbNR is the spin-splittings of 62 and $44 \mathrm{meV}$, respectively, at the VBM and $C B M$. The imbalanced charge distributions enable the electric modulation of bands around the Fermi level in a W-aSbNR. The in-plane electric field across the edges modifies the spatially separated localized states. As a result, the SOC-induced band inversion and electron-hole pockets with appropriately the same size appear. The unusual electronic properties predicted in a W-zSbNR provide another platform to exploit and understand the XMR effect.

\section{METHODS}

The calculations are performed using the density functional theory in conjunction with the projector augmented wave potentials ${ }^{45}$ and PBE generalized gradient approximation ${ }^{46}$ to the electronic exchange and correlation as implemented in the Vienna ab initio simulation package (VASP) code. ${ }^{47,48}$ The Monkhorst-Pack $k$-point sets $1 \times 11 \times 3$ for aWaSbNR, $1 \times 7 \times 3$ for aW-zSbNR, B-aSbNR, and B-zSbNR are used. The energy cutoff for the plane-wave basis set is $500 \mathrm{eV}$. In all, $10^{-5} \mathrm{eV}$ and $10^{-2} \mathrm{eV} / \AA$ are the criteria of energy and force convergence. Sufficient vacuum layers of $15 \AA$ perpendicular to the plane and $20 \AA$ along the ribbon width are adopted to avoid the interactions between the periodic images. In order to simulate the influence of in-plane electric field using VASP, an artificial dipole layer in the middle of the vacuum in the $x$ direction (Fig. 1) is added.

\section{ACKNOWLEDGEMENTS}

This work is supported by National Natural Science Foundation of China (51671142 and U1632152), Key Project of Natural Science Foundation of Tianjin City (16JCZDJC37300 and 14JCZDJC37800), Program for New Century Excellent Talents in University (NCET-13-0409). It is also supported by High Performance Computing Center of Tianjin University, China

\section{AUTHOR CONTRIBUTIONS}

W. B. M. and Y. S. designed the project. Y. S. performed the simulations, analyzed the data and wrote the manuscript. W. B. M. and X. C. W. gave detailed discussions and revised the manuscript.

\section{COMPETING INTERESTS}

The authors declare no conflict of interest.

\section{REFERENCES}

1. Geim, A. K. \& Novoselov, K. S. The rise of graphene. Nat. Mater. 6, 183-191 (2007).

2. Novoselov, K. S. et al Two-dimensional gas of massless Dirac fermions in graphene. Nature 438, 197-200 (2005).

3. Dean, C. R. et al. Boron nitride substrates for high-quality graphene electronics. Nat. Nanotechnol. 5, 722-726 (2010).

4. Lu, G. et al. Synthesis of large single-crystal hexagonal boron nitride grains on $\mathrm{Cu}$ Ni alloy. Nat. Commun. 6, 6160 (2015).

5. Yin, J. et al. Aligned growth of hexagonal boron nitride monolayer on germanium. Small 11, 5375-5380 (2015).

6. Yin, J. et al. Large single-crystal hexagonal boron nitride monolayer domains with controlled morphology and straight merging boundaries. Small 11, 4497-4502 (2015).

7. Ganatra, R. \& Zhang, Q. Few-layer $\mathrm{MoS}_{2}$ : A promising layered semiconductor. ACS Nano 8, 4074-4099 (2014).

8. Radisavljevic, B., Whitwick, M. B. \& Kis, A. Integrated circuits and logic operations based on single-layer MoS $_{2}$. ACS Nano 5, 9934-9938 (2011).

9. Late, D. J., Liu, B., Ramakrishna Matte, H. S. S., Dravid, V. P. \& Rao, C. N. R. Hysteresis in single-layer $\mathrm{MoS}_{2}$ field effect transistors. ACS Nano 6, 5635-5641 (2012).

10. Mak, K. F., Lee, C., Hone, J., Shan, J. \& Heinz, T. F. Atomically thin $\operatorname{MoS}_{2}$ : A new direct-gap semiconductor. Phys. Rev. Lett. 105, 136805 (2010).

11. Zhu, Z. Y., Cheng, Y. C. \& Schwingenschlögl, U. Giant spin-orbit-induced spin splitting in two-dimensional transition-metal dichalcogenide semiconductors. Phys. Rev. B 84, 153402 (2011).

12. Zhang, S. et al. Semiconducting group 15 monolayers: A broad range of band gaps and high carrier mobilities. Angew. Chem. Int. Ed. 55, 1666-1669 (2016).

13. Yan, Q. et al. Intrinsic current-voltage characteristics of graphene nanoribbon transistors and effect of edge doping. Nano Lett. 7, 1469-1473 (2007).

14. Huang, B. et al. Making a field effect transistor on a single graphene nanoribbon by selective doping. Appl. Phys. Lett. 91, 253122 (2007). 
15. Wang, X. et al. Room-temperature all-semiconducting sub-10-nm graphene nanoribbon field-effect transistors. Phys. Rev. Lett. 100, 206803 (2008).

16. Chitara, B., Panchakarla, L. S., Krupanidhi, S. B. \& Rao, C. N. R. Infrared photo detectors based on reduced graphene oxide and graphene nanoribbons. Adv. Mater. 23, 5419-5424 (2011).

17. Zhang, Z., Chen, C. \& Guo, W. Magnetoelectric effect in graphene nanoribbons on substrates via electric bias control of exchange splitting. Phys. Rev. Lett. 103, 187204 (2009).

18. Sahu, B., Min, H., MacDonald, A. H. \& Banerjee, S. K. Energy gaps, magnetism, and electric-field effects in bilayer graphene nanoribbons. Phys. Rev. B 78, 045404 (2008).

19. Son, Y. W., Cohen, M. L. \& Louie, S. G. Half-metallic graphene nanoribbons. Nature 444, 347-349 (2006).

20. Kan, E., Li, Z., Yang, J. \& Hou, J. G. Half-metallicity in edge-modified zigzag graphene nanoribbons. J. Am. Chem. Soc. 130, 4224-4225 (2008).

21. Zheng, F. et al. Half metallicity along the edge of zigzag boron nitride nanoribbons. Phys. Rev. B 78, 205415 (2008).

22. Pan, H. \& Zhang, Y. W. Edge-dependent structural, electronic and magnetic properties of $\mathrm{MoS}_{2}$ nanoribbons. J. Mater. Chem. 22, 7280 (2012).

23. Tsai, H. S. et al. Direct synthesis and practical bandgap estimation of multilayer arsenene nanoribbons. Chem. Mater. 28, 425-429 (2016).

24. Tsai, H. S., Chen, C. W., Hsiao, C. H., Ouyang, H. \& Liang, J. H. The advent of multilayer antimonene nanoribbons with room temperature orange light emission. Chem. Commun. 52, 8409-8412 (2016).

25. Aktürk, O. Ü., Özçelik, V. O. \& Ciraci, S. Single-layer crystalline phases of antimony: Antimonenes. Phys. Rev. B 91, 235446 (2015).

26. Ali, M. N. et al. Large, non-saturating magnetoresistance in $\mathrm{WTe}_{2}$. Nature 514, 205 (2014).

27. Wu, X., Zhang, X., Wang, X. \& Zeng, Z. Spin density waves predicted in zigzag puckered phosphorene, arsenene and antimonene nanoribbons. AlP Advances 6 , 045318 (2016)

28. Zhang, S. et al. Semiconductor-topological insulator transition of twodimensional SbAs induced by biaxial tensile strain. Phys. Rev. B 93, 245303 (2016).

29. Zeng, L. K. et al. Compensated semimetal LaSb with unsaturated magnetoresistance. Phys. Rev. Lett. 117, 127204 (2016).

30. Wang, K., Graf, D., Li, L., Wang, L. \& Petrovic, C. Anisotropic giant magnetoresistance in $\mathrm{NbSb}_{2}$. Sci. Rep. 4, 7328 (2014).

31. Shen, B., Deng, X. Y., Kotliar, G. \& Ni, N. Fermi surface topology and negative longitudinal magnetoresistance observed in the semimetal $\mathrm{NbAs}_{2}$. Phys. Rev. $B$ 93, 195119 (2016).

32. Wang, Y. Y., Yu, Q. H., Guo, P. J., Liu, K. \& Xia, T. L. Resistivity plateau and extremely large magnetoresistance in $\mathrm{NbAs}_{2}$ and TaAs. Phys. Rev. B 94, 041103 (2016).

33. Tafti, F. F., Gibson, Q. D., Kushwaha, S. K., Haldolaarachchige, N. \& Cava, R. J. Resistivity plateau and extreme magnetoresistance in LaSb. Nat. Phys 12, 272-277 (2015)
34. Wang, X. F. et al. Quantum electronics: evidence of both surface and bulk Dirac bands and anisotropic nonsaturating magnetoresistance in $\mathrm{ZrSiS}$. Adv. Electron. Mater. doi:10.1002/aelm.201670055 (2016).

35. Jiang, J. et al. Signature of strong spin-orbital coupling in the large nonsaturating magnetoresistance material WTe 2 . Phys. Rev. Lett. 115, 166601 (2015).

36. Pletikosić, I., Ali, M. N., Fedorov, A. V., Cava, R. J. \& Valla, T. Electronic structure basis for the extraordinary magnetoresistance in WTe $\mathrm{W}_{2}$. Phys. Rev. Lett. 113, 216601 (2014).

37. Cai, P. L. et al. Drastic pressure effect on the extremely large magnetoresistance in WTe $e_{2}$ : Quantum oscillation study. Phys. Rev. Lett. 115, 057202 (2015).

38. Flynn, S., Ali, M. \& Cava, R. J. The effect of dopants on the magnetoresistance of $W T e_{2}$. arXiv: 1506.07069.

39. Wang, Y. L., Wang, K. F., Reutt-Robey, J., Paglione, J. \& Fuhrer, M. S. Breakdown of compensation and persistence of nonsaturating magnetoresistance in gated $\mathrm{WTe}_{2}$ thin flakes. Phys. Rev. B 93, 21108 (2016).

40. Rhodes, D. et al. Role of spin-orbit coupling and evolution of the electronic structure of $\mathrm{WTe}_{2}$ under an external magnetic field. Phys. Rev. B 92, 125152 (2015).

41. Du, X., Tsai, S. W., Maslov, D. L. \& Hebard, A. F. Metal-insulator-like behavior in semimetallic bismuth and graphite. Phys. Rev. Lett. 94, 166601 (2005).

42. Das, P. K. et al. Layer-dependent quantum cooperation of electron and hole states in the anomalous semimetal WTe 2. Nat. Commun. 7, 10847 (2016).

43. Ouyang, F. P. et al. Hydrogenation-induced edge magnetization in armchair $\mathrm{MoS}_{2}$ nanoribbon and electric field effects. Appl. Phys. Lett. 104, 071901 (2014).

44. Kou, L. Z. et al. Tuning magnetism and electronic phase transitions by strain and electric field in zigzag $\mathrm{MoS}_{2}$ nanoribbons. J. Phys. Chem. Lett. 3, 2934-2941 (2012).

45. Blöchl, P. E. Projector augmented-wave method. Phys. Rev. B 50, 17953 (1994).

46. Perdew, J. P., Burke, K. \& Ernzerhof, M. Generalized gradient approximation made simple. Phys. Rev. Lett. 77, 3865 (1996).

47. Kresse, G. \& Furthmüller, J. Efficient iterative schemes for ab initio total-energy calculations using a plane-wave basis set. Phys. Rev. B 54, 11169 (1996).

48. Kresse, G. \& Joubert, D. From ultrasoft pseudopotentials to the projector augmented-wave method. Phys. Rev. B 59, 1758 (1999).

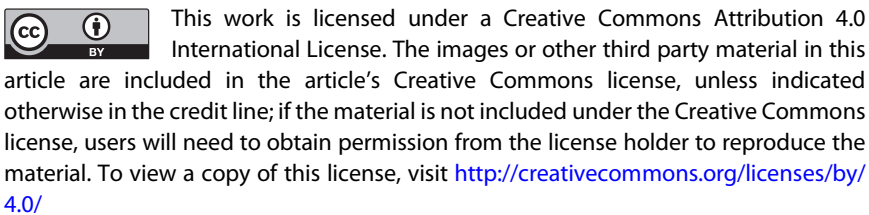
$4.0 /$

(c) The Author(s) 2017

Supplementary Information accompanies the paper on the npj Quantum Materials website (doi:10.1038/s41535-017-0022-y). 